\title{
Value-added interregional inequality assessment of border regions in Russia
}

\author{
Natalya S. Epifanova ${ }^{1 *}$ \\ ${ }^{1}$ Novosibirsk State University of Economic and Management, Department of Regional Economy and \\ Public Administration, 56 Kamenskaya Str., Novosibirsk, 630099, Russia
}

\begin{abstract}
The economic development of Russian border regions is accompanied by a high level of interregional disparities both within the group of border regions and across the country. The purpose of the article is to assess the degree of interregional inequality of border regions in comparison with inner ones. Based on the key indicator of regional economic development, Gross Regional Product (GRP) per capita, various indicators of interregional inequality (coefficients of variation, the ratio of the maximum value to the minimum value, the Gini and Theil coefficients) were calculated, and $\sigma$-convergence and absolute $\beta$-convergence were estimated. Estimations of all indicators show that sharp changes in inequality, as well as the level of interregional inequality itself, are characteristic of border regions. For the interior regions, the dynamics are smoother, and the degree of inequality is significantly lower. This allows concluding that the main reason for the formation of interregional inequalities in Russia is inter-regional inequality in the group of border regions.
\end{abstract}

Keywords: border regions, regional disparities, economic convergence, Russian regions

\section{Introduction}

The economic development of border regions in modern conditions is of particular importance. Due to the constantly growing geopolitical risks and threats, the border regions are the first to become a kind of a buffer for the macroeconomics as a whole: the indicators of their economic development are the first to reflect the influence of these negative factors. It is likely that the reflection of the problem of interregional inequality in Russia's economy thus will correspond to a high-er level of interregional differentiation specifically for border regions (in comparison with internal ones). The purpose of this paper is to test the hypothesis of whether the level of interregional inequality is higher for the Russian border regions in comparison to the inner regions. Confirmation of this hypothesis should become an important landmark in the reorientation of the regional policy of the federal center, aimed at reducing interregional inequality in Russia.

* Corresponding author: nucifraga@mail.ru 


\section{Literature review}

There are quite a few studies devoted to the issue of border regions. But there is no monosemantic approach to the definition of one. The most popular definition was proposed by Granberg, who classifies all border regions as problematic and defines them as territories that experience "a significant influence of the state border, the main functions of which are barrier, filtering, contact" [1, p. 332]. This approach to the definition of border regions suggests the presence of an additional and special potential for international cooperation.

The peripheral position of border regions is considered by many authors as their specific characteristic [2;3]. Research on regional economics has focused on how border regions can overcome location disadvantages arising from barriers created by national borders and turn this circumstance into a competitive advantage through cross-border relations and cooperation with neighboring states.

In the same way, many authors consider a border region as a territory that has several specific features. But most of these authors define these features differently. Some authors interpret border regions as territories whose economic development is largely determined by the nature of a specific border and the role they play in international economic relations [4; 5]. Based on this approach, researchers pay special attention to the border relations of this region with the territories of the neighboring state.

In the literature devoted to assessing interregional inequalities, there is a sufficient variety of concepts and approaches to assessing spatial inequalities in eco-nomic development, which is caused by the significant urgency of this problem. In the current study, the author chooses an approach based on standard indica-tors for assessing regional differentiation: the coefficient of variation, the ratio of the maximum value of the indicator to the minimum, as well as entropy coefficients to the assessment of the uneven distribution of the regional product flows. Due to our purposes, the theory of convergence was also applied, which was used by the author in the interpretation of the neoclassical theory of growth [6]. This theory defines the basic model for convergence of unconditional $\beta$-convergence and $\sigma$-convergence.

\section{Materials and Methods}

The present research consists of two parts. The first one is the study of the ratio of the maximum value of Gross Regional Product (GRP) per capita to the minimum and of the coefficient of variation and in the corresponding groups of Russian regions. All data on GRP per capita of Russian regions for the period of 1996-2019 are divided according to three main groups of regions: the first group includes border regions (43 constituent entities of the Russian Federation except for the Republic of Crimea and Sevastopol, which are excluded from the analysis, since data on them are available only for a short period of time); the second group contains all internal regions (37 regions); the third group comprises all internal regions excluding Moscow (since Moscow usually has a significant gap in the average regional value in all economic indicators of the regional development in Russia).

Based on the existing features of regional statistics and changes in the Russian territorial division in the period from 1996 to 2019, the regions including the autonomous okrugs are considered as a whole, the okrugs were not considered separately. Comparative analysis for the three groups of regions of the dynamics of the coefficient of variation and the ratio of the maximum value of GRP to the minimum will make it possible to assess which of these groups is more heavily burdened by interregional inequality.

To calculate the coefficient of variation, the formula for the ratio of the standard (rootmean-square) deviation to the mean value was used: 


$$
\sigma=\frac{\sqrt{\frac{1}{n} \sum_{i=1}^{n}\left(G R P_{i}-G R P_{a v}\right)^{2}}}{G R P_{a v}}
$$

where $\sigma$ is the coefficient of simple variation; GRPi is the value of GRP per capita for the region i; GRPav is the regional average value of GRP in each group of regions; $n$ is the number of regions in the group for which the coefficient of variation is calculated.

To confirm $\sigma$-convergence, it is necessary to fulfill the condition of reducing the variance of the level of per capita GRP during the period under consideration. To assess $\sigma$ convergence, one can use indicators of variance, standard deviation, and coefficient of variation. Regression analysis is commonly used to assess regional $\beta$-convergence. Within the framework of this study, the approach of "club" divergence was used, namely, the absolute convergence was assessed for the three main groups of regions, identified in accordance with the purpose of the work. In particular, for each group, a regression equation of the form was constructed:

$$
\left(\frac{G R P_{T}}{G R P_{0}}\right)^{\frac{1}{T}}=\beta_{0}+\beta_{1}^{*} \ln G R P_{0}+\varepsilon
$$

where $\mathrm{GRP}_{0}$ and $\mathrm{GRP}_{\mathrm{T}}$ are the values of the region's per capita GRP at the initial and final moments of the considered time interval; $\left(\frac{G R P_{T}}{G R P_{0}}\right)^{\frac{1}{T}}$ is the average growth rate of the region's GRP per capita; $\beta_{0}$ is a constant; $\beta_{1}$ is the regression coefficient; $T$ is the number of time intervals. This equation tests for a negative correlation between the rates of economic growth and the initial level of regional economic development, therefore, if $\beta_{1}<0$ in this equation, then the hypothesis of absolute $\beta$-convergence is accepted.

The second direction of this study contains calculations and assessment of interregional differentiation of border regions in comparison with internal ones based on the entropy approach [7]. For the purposes of this study, such an approach allowed selecting two entropy coefficients. These are Theil and Gini coefficients. Both have an important advantage from the perspective of this study, since they allow assessing inequality by means of the ratio of the distribution of a feature in populations with different numbers of units. Another important argument in favor of the choice of these coefficients is that the result obtained when calculating them is not distorted depending on the scale of regional economies, the differences of which are significant for Russian regions.

Theil's coefficient is calculated according to the formula:

$$
T=\frac{1}{n} * \sum_{i=1}^{n}\left(\frac{G R P_{i}}{G R P_{a v}} * \ln \frac{G R P_{i}}{G R P_{a v}}\right)
$$

where $T$ is the Theil coefficient; $G_{R P}$ is the value of GRP per capita of the $i$-th region; GRP $_{a v}$ is the regional average value of GRP in each group of regions; $n$ is the number of regions in the group for which the coefficient of Theil is calculated.

Theil's coefficient, therefore, is a measure of the territorial dispersion of GRP per capita. To calculate the Gini coefficient in each of the three groups of regions, four equal groups in the number of regions were singled out after ordering the entire series within the group by the value of GRP per capita. Further, the coefficient is calculated as follows:

$$
K=1-2 * \sum_{i=1}^{n} x_{i}^{*} \text { cumGRP }+\sum_{i=1}^{n} x_{i}^{*} G R P_{i}
$$

where $K$ is the Gini coefficient; $x_{i}$ is the share of regions of the $\mathrm{i}$-th group $\left(i=\overline{1, n}, \sum_{i=1}^{n} x_{i}=1\right)$ ; cumGRP $_{i}$ is the cumulative share of GRP of the $\mathrm{i}$-th and previous groups of regions, that is, 
the share of GRP falling on the regions from the 1 st to the $\mathrm{i}$-th inclusive; $G R P_{i}$ is the share of the GRP of the $\mathrm{i}$-th group of regions in the total GRP of the country $\left(i=\overline{1, n}, \sum_{i=1}^{n} y_{i}=1\right) ; n$ is the number of regions in the group of regions for which the coefficient is calculated.

The closer the value obtained in the calculations to 1, the higher the degree of uneven distribution of GRP between regions. The closer this value is to 0 , the more evenly the GRP is distributed between the regions.

We also note that it is incorrect to draw conclusions about the degree of interregional inequality based just on these coefficients. In this study they are used to assess the degree of inequality in the level of economic development of Russian regions as subsidiary tools for such an assessment. It is generally accepted that both coefficients assess ceteris paribus the grade of inequality rather qualitatively, as they have such characteristics as independence from the number of observations and from the mean value of the distribution, symmetry, and sensitivity to redistribution [7]

\section{Results}

Estimations of the coefficient of variation for groups of border regions, inner regions and inner regions excluding Moscow (Fig. 1) showed that the variation across regions of the GRP value relative to the average value for each group of regions on average for border regions is two or more times higher than the specified calculated parameter in the group of inner territories. Interestingly, for the inner regions excluding Moscow, the coefficient of variation in the period between 1996 and 2019 fluctuates in the range from 39 to 48\%. Although it indicates some heterogeneity of these regions, this heterogeneity is significantly lower compared to the border regions, for which the coefficient of variation turned out to be extremely high and varied from 73 to $100 \%$ in different time periods. The dynamics of the coefficient of variation for inner regions excluding Moscow after 2005 tends to gradually decrease. Some researchers interpret similar reduction as an emerging trend towards convergence in the level of economic development of regions (see, for example: [8-10]). However, starting from 2014, a gradual increase in the coefficient of variation has also been observed in the group of inland regions.

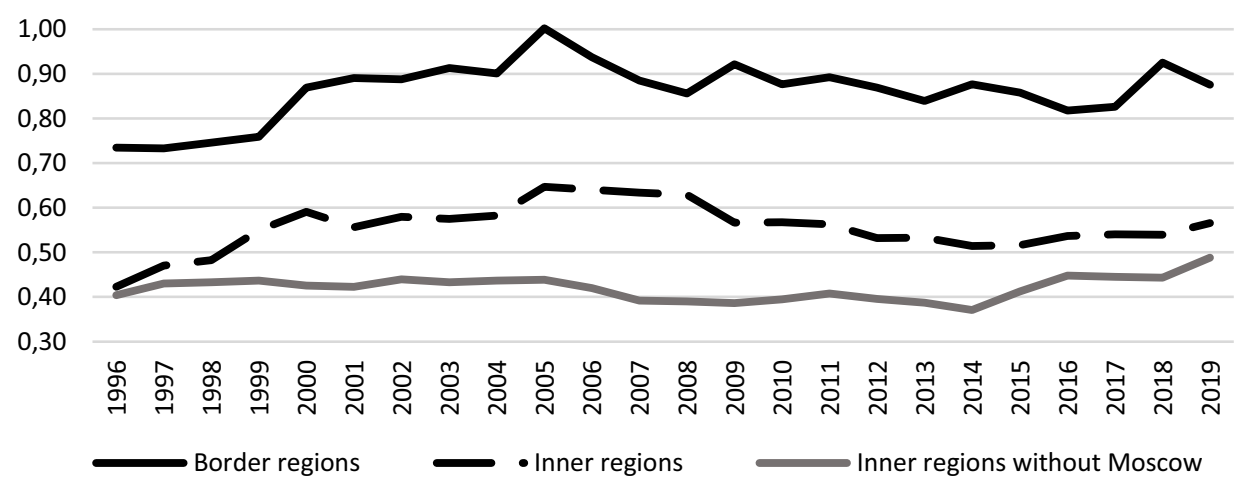

Fig. 1. Dynamics of the coefficient of variation calculated by the value of GRP per capita for the period from 1996 to 2019.

The ratio of the maximum GRP per capita to the minimum for the three groups of Russian regions (Fig. 2) also demonstrates a significantly higher value for the group of border regions. According to the estimating result, the disparities grade of GRP per capita throughout the 
entire period from 1996 to 2019 was three or more times higher in comparison with the inner regions.

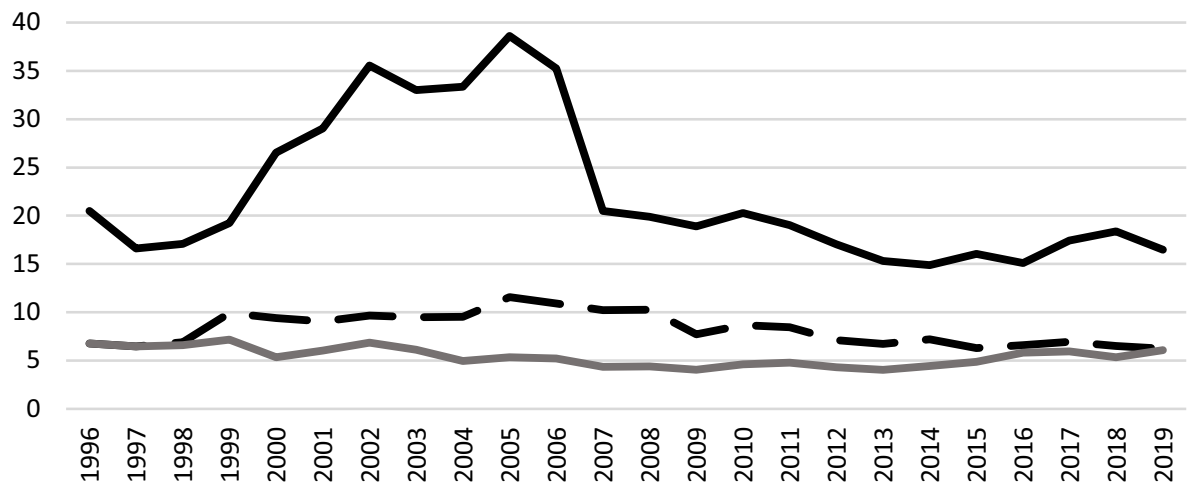

$\longrightarrow$ Border regions $\quad$-Inner regions Inner regions without Moscow

Fig. 2. Dynamics of the ratio of the maximum to the minimum value of GRP per capita for the period from 1996 to 2019.

The $\beta$-convergence hypothesis is the assumption that relatively underdeveloped regions grow faster than economically developed ones, which means that the depth of interregional inequality decreases over time. This hypothesis was also not confirmed when calculating the coefficients of the regression equations, which are almost the same for the three groups of regions.

The Theil coefficient for border and inland regions shows that the level of interregional differentiation of GRP per capita for border regions is significantly higher than that for inland regions (Fig. 3). Moreover, if we consider the inner regions without Moscow, then according to the Theil coefficient, the level of interregional inequality is incredibly low in them and approaches zero in some periods of time.

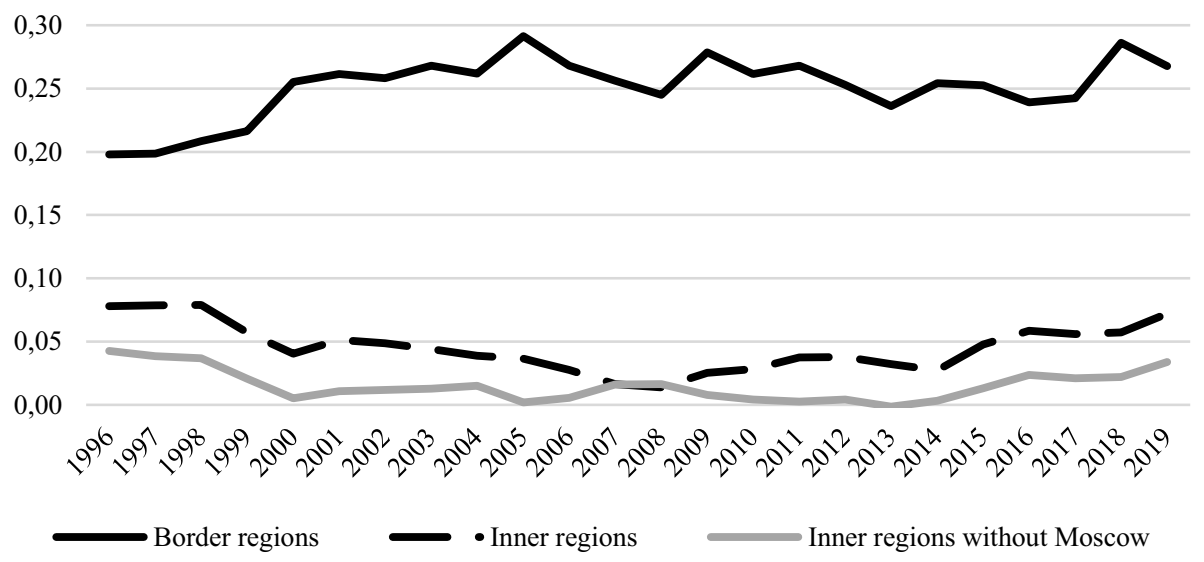

Fig. 3. Dynamics of the Theil coefficient calculated on the basis of GRP per capita for the period from 1996 to 2019.

Another aspect of inequality can be represented using the coefficient of heterogeneity of regional development, which can be calculated as the entropy coefficient. This is the Gini 
coefficient. The estimation result for the Gini coefficient based on GRP per capita reflects the spatial concentration of GRP per capita and the grade of interregional disparities in the sense of uneven concentration of per capita gross product in the group of the richest regions.

Figure 4 shows the dynamics of the Gini coefficient. Inequality between regions in all three groups gradually increased over the period from 1996 to 2019, but concomitantly it remained at a relatively higher level for border regions. In recent years, there has been a faster growth in interregional inequality in the group of inner regions excluding Moscow, but inequality among border regions is also growing. Although growth among border regions is slower, it remains at a higher level in comparison with inner regions.

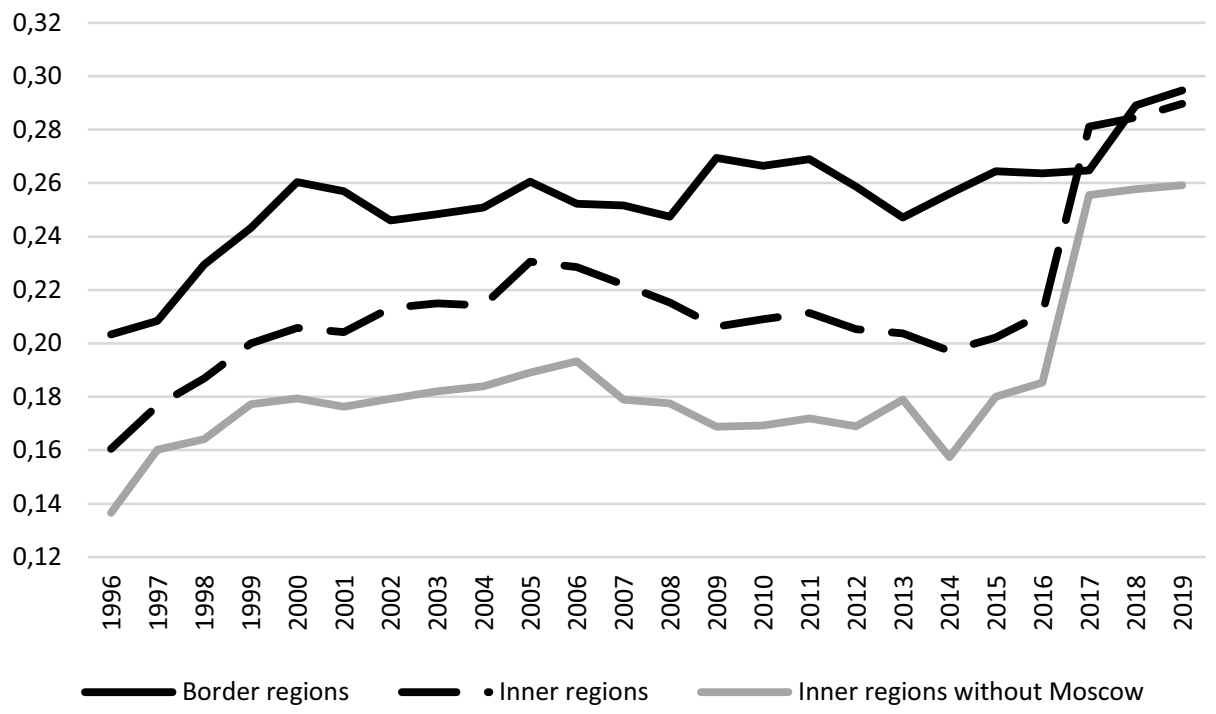

Fig. 4. Dynamics of the Gini coefficient on the value of GRP per capita for the period from 1996 to 2019.

\section{Discussion}

The inconsistency of the estimates of the regression coefficients when assessing the absolute $\beta$-convergence is a sign of the absence of a single path of proportional growth both among internal and among border regions. Further study of the problem of interregional inequality in the group of border regions is necessary considering the problem of the influence of the economic development specifics by the territories of neighboring countries to the regional economic development in the countries. It is also necessary to assess the conditional $\beta$ convergence, which would take into account additional factors that may affect the economic development of regional economies. We believe, such additional factors of the economic development of border regions should include the influence of the state border that its position has on the economy of a given region, as well as the region's involvement in international trade.

Since many researchers note that the border regions of one country form a common economic space with similar territories of the neighboring country, it is likely that one of the factors of inequality in the group of border regions is the influence of the border territories of neighboring countries. This influence is exerted not only by the comparative advantages of neighboring territories, but also by the structural features of their regional economies, as well as the peculiarities of the national policies of neighboring countries in relation to their border areas. The further direction of this study should be to test this assumption, which will 
probably help explain why the levels of economic development of the Russian borderlands differ so much in the European part and in the Far-Eastern territory.

For example, Russia and China exercise a relatively liberal approach to the implementation of cross-border cooperation strategies on the part of Russia and active measures of the Chinese government to support their border provinces. As a result, differences in the approaches lead to a decrease in the efficiency of cross-border interaction and the lag of Russian border regions from the average Russian level. The consequence is that cross-border interaction between the regions of Russia and China is built mainly on trade and export of labor resources from China to Russia. Thus, China gets additional trade channels and confidently growing sales markets for consumer goods and gets sources of raw materials and primary products. Since for Russian regions bordering with China, cooperation with the neighboring territories is important precisely as a compensatory mechanism for minimizing transport costs amid a continuous increase in transport tariffs within Russia and, as a result, weakening economic ties with the internal Russian regions, the lack of adequate regional policy of the Russian federal center, with the active support of its border regions of China's regional policy, leads to the conservation of the raw material specialization for Russian border regions in the Far East. Therefore, interregional inequality in Russia is increasing.

\section{Conclusion}

The study shows that interregional differentiation in Russia is formed mainly due to inequality between Russian border regions. At the same time, the dynamics of almost all calculated indicators of inequality for the inner regions is rather smooth, without sharp fluctuations, and for the border regions it is more abrupt in nature. This result suggests that border regions play a special role in the formation of spatial disparities in Russia, and the existing regional policy of the federal center does not consider this important feature.

Since this study used the so-called modified version of the Theil index, which allows one to take into account the different scale of regions [14], it is believed that this index more accurately reflects the inequality in the distribution of per capita gross product between regions, so it is interesting that the calculations of the Theil index did not show an increase in interregional inequality for the group of internal regions in 2005, which is very clear for all the other calculated indicators of interregional inequality. At the same time, calculations of the Theil index for border regions, as well as calculations of other indicators, a high increase in interregional inequality in 2005. This suggests that interregional inequality in the Russian Federation in 2005 increased to a large extent precisely due to the border regions.

\section{References}

1. A.G. Granberg, Fundamentals of the regional economy (High School of Economics, Moscow, 2001)

2. S.S. Artobolevsky, L.B. Vardomsky, Problems of social geography, 2, 14-26 (2010)

3. D.D. Tsyrenov, M.P. Boksha, Bulletin of the Buryat State University. Economics and Management, 1, 62-72 (2020)

4. M. Brülhart, O. Cadot, A. Himbert, Fondation pour les études et recherches sur le développement international, Working Paper No. 251 (2019)

5. P. Ulrich, S. Troitsky, J. Frontier Studies, 4-2, 16, 234-256 (2019)

6. R.J. Barro, X. Sala-i-Martin, Economic Growth (McGraw Hill, New York, 1995)

7. S.P. Chakravarty, A. Majumder, Australian Economic Papers, 40, 1, 1-13 (2001) 
8. M.Y. Malkina, Spatial Economics, 3, 44-66 (2014)

9. E.A. Kolomak, Interexpo Geo-Siberia, 3, 1, 88-95 (2020)

10. M.V. Moroshkina, Economic analysis: theory and practice, 45, 396, 20-28 (2014) 